\title{
Hall Current Effect in MHD Oscillatory Couple Stress Dusty Fluid through an Inclined Saturated Permeable Channel
}

\author{
A. Govindarajan ${ }^{1}$, R. Vijayalakshmi ${ }^{2}$ * \\ ${ }^{I}$ Department of Mathematics, SRM IST, Kattankulathur. \\ ${ }^{2}$ Department of Mathematics, SRM IST, Ramapuram. \\ *Correspondence address: vijayalakshmi.r@rmp.srmuniv.ac.in
}

\begin{abstract}
This paper aims to study the Hall current effect in MHD (Magneto Hydro Dynamics) oscillatory couple stress dusty fluid (blood) flowing through an inclined permeable medium channel. A mathematical model for both the fluid and particle phases are framed based on the conservation laws. The exact form of solutions for velocity, energy, species concentration profiles are got by solving the corresponding partial differential equations. To have a better knowledge about the problem, quantitative estimates are presented pictorially and discussed to know the main features of different measurable factors like radiation, Hall current, Schmidt number, Nusselt number etc.
\end{abstract}

Keywords: biomedical systems; Hall current; couple stress; dusty fluid; blood; MHD; oscillatory flow; inclined channel

\section{Introduction}

A dusty fluid is a fluid with suspension particles. The idea behind dusty fluid flow is a subject of interest for researchers, since this type of flow occurs in industrial filtration, powder manufacturing industry, transport of liquid waste materials in chemical processing, production of plastic products, purification of crude oil, pulp and paper industry, environment pollution, smoke emission from vehicles, nuclear process and in different geophysical situations.

The subject of couple stress fluid is very useful in understanding many real life problems because it possesses the mechanism to describe rheological complex fluids such as liquid crystals and human blood. Couple stress fluid is a special kind of nonNewtonian fluids. Further research shows that many authors have assumed blood to be a suspension of spherical rigid particles (red cells) and this suspension of spherical rigid particles will give rise to couple stresses in a fluid.

Magnetic field has retarding influence on the fluid velocity and the angle of inclination of magnetic field has accelerating influence on the fluid velocity.

When an electrical current passes through a fluid placed in a magnetic field, a potential directly proportional to the current and to the magnetic field is produced across the fluid. Its direction is perpendicular to both the current and to the magnetic field. This effect is known as the Hall Effect, and it has many practical applications in many devices such as magnetic field measurements and position and motion detectors.

Saffman [1] was the first to propose equations of motion for a mixture of fluid and dust particles. Varvara et. al. [2] modelled the blood as two-phase dusty fluid. Makinde [3] made an examination on heat transfer effects to MHD oscillatory fluid through a permeable medium channel. Stokes [4] made a detailed study of couple stresses in fluids.

Kulshretha et. al. [5] contemplated the wave structure in oscillatory Couette stream of a dusty gas. The heat transfer effects in an unsteady dusty fluid flow through a symmetric channel was analyzed by Gireesha et. al. [6]. Makinde [7] discussed the heat transfer effects and Navier slip condition by varying the physical properties of dusty fluid. Prakash et. al. [8] investigated the MHD flow of dusty viscoelastic gas through a partially infinite plate. Govindarajan et. al. [9] examined the mass transfer effects of MHD oscillatory flow in a permeable medium channel. Vidhya et. al. [10] concentrated in the transfer of mass effects of MHD oscillatory flow through a asymmetric channel. Navier slip effect on radiative MHD oscillatory flow with chemical reaction in an asymmetric channel was studied by Govindarajan et. al. [11] . The problem of chemically reacting MHD oscillatory Navier slip flow in an asymmetric channel with both varying concentration and temperature was solved by Govindarajan et. al. [12]. Om Prakash et. al. [13] studied the heat transfer effects to MHD oscillatory dusty fluid flow in a permeable medium channel. Ogulu [14] gave the relation for radiative heat flux. Govindarajan et. al. [15] studied the effects of heat and mass transfer to MHD oscillatory dusty fluid flow in a permeable channel. Effect of Hall current in oscillatory flow of a couple stress fluid in an inclined channel was discussed by Nirmala et. al.[16]. Pandurangan et. al. [17] concentrated in Hall effects and rotation effects on MHD flow past an exponentially accelerated vertical plate with heat and mass transfer. Dulal Pal [18] analyzed the influence of Hall current and Thermal radiation on MHD convective heat and mass transfer in a rotating permeable channel with chemical reaction. Singh et. al. [19] investigated the Hydromagnetic oscillatory flow of dusty fluid in a rotating permeable channel. Chand et. al. [20] examined the effects of Hall current and rotation on chemically reacting and radiating MHD oscillatory dusty viscoelastic flow through permeable vertical channel.

In this current paper, the blood is modeled by a couple stress dusty fluid and we investigate the effect of hall current in MHD oscillatory flow in an inclined saturated permeable medium channel. The temperature and species concentration along the walls of the channel are not same. The mathematical equations are solved us- 
ing the ordinary differential equation method and the boundary conditions are applied. The effect of different physical parameters on velocity, energy and species concentration profiles has been studied and numerical results obtained are presented graphically. The model is defined numerically in Part 2. The mathematical solution is given in Part 3. The relevant ends are delineated pictorially and examined in Part 4 . The final comments are given in Part 5.

\section{Description of the Problem}

An incompressible, viscous, electrically conducting, heat generating, optically thin and chemically reacting couple stress dusty fluid flowing through an inclined permeable channel is considered The inclined channel is under the influence of an externally applied uniform magnetic field $\mathrm{B}_{0}$ and radiative heat transfer as shown in Fig. 1.

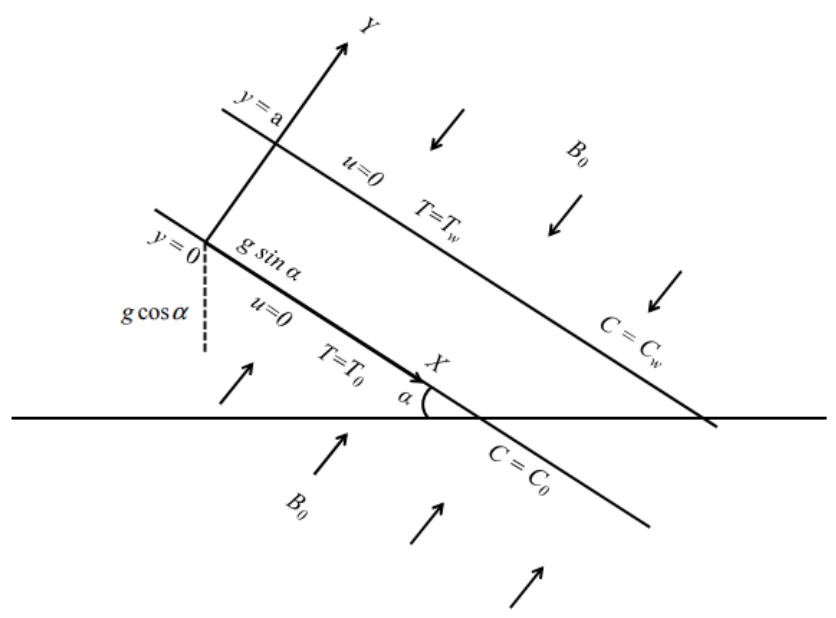

Fig. 1: Physical Configuration of the problem

The suspension particles are solid, round in shape. They are nonconductive equal in size and spreads equally in the flow region and their number density $\mathrm{N}_{0}$ is constant throughout the motion The amount of heat transfer between the dust particles is same throughout the motion. The collisions between the suspension particles and mass transfer are also considered. The Reynolds number is taken to be very small so that induced magnetic field can be avoided. Hence the fluid moving region has same temperature and magnetic field. The suspension particles are uniformly distributed and transported in the fluid such that the continuity equation is satisfied.

Governing Equations: Based on Boussinesq assumptions for an incompressible couple stress dusty fluid model, the mathematical equations are based on the conservation laws of mass, linear momentum for both fluid and suspension particles phases. Let $u^{*}\left(u^{*}, v^{*}, w^{*}\right)$ and $u_{p}{ }^{*}\left(u_{p}{ }^{*}, v_{p}{ }^{*}, w_{p}{ }^{*}\right)$ be the fluid and particle velocity respectively. The momentum, energy and species equations are considered in the form

$$
\begin{aligned}
\frac{\partial u^{*}}{\partial t^{*}}=- & \frac{1}{\rho} \frac{\partial P^{*}}{\partial x^{*}}+v \frac{\partial^{2} u^{*}}{\partial y^{* 2}}+\eta \frac{\partial^{4} u^{*}}{\partial y^{* 4}}-\frac{v}{k^{*}} u^{*}+\frac{N_{0} K_{0}}{\rho}\left(u p^{*}-u^{*}\right) \\
& -\frac{\sigma B_{0} u^{*}}{\rho\left(1+m^{2}\right)}+g \beta\left(T^{*}-T_{0}\right) \sin \alpha+g \beta *\left(C^{*}-C_{0}\right) \sin \alpha \\
-\frac{1}{\rho} P^{* \prime}\left(y^{*}\right)=0 & \\
\frac{\partial u_{p}^{*}}{\partial t^{*}}= & K_{0}\left(u^{*}-u_{p}^{*}\right) \\
\frac{\partial T^{*}}{\partial t^{*}}= & \frac{K^{*}}{\rho C_{p}} \frac{\partial^{2} T^{*}}{\partial y^{* 2}}-\frac{1}{\rho C_{P}} \frac{\partial q}{\partial y^{*}}+\frac{Q^{*}}{\rho C_{P}}\left(T^{*}-T_{\mathrm{O}}\right)
\end{aligned}
$$

$\frac{\partial C^{*}}{\partial t^{*}}=D_{m} \frac{\partial^{2} C^{*}}{\partial y^{* 2}}-K_{c}^{* *}\left(C^{*}-C_{0}\right)$

where $m=\frac{\sigma B_{0}}{e N_{m_{0}}}$ is the Hall parameter, e is the electric charge,

$N_{m_{0}}$ is the electrons' number density.

The boundary conditions are

$$
\begin{aligned}
& u^{*}=u_{\mathrm{p}}^{*}=0, \mathrm{~T}^{*}=\mathrm{T}_{\mathrm{w}}, \mathrm{C}^{*}=\mathrm{C}_{\mathrm{w}}, \frac{\partial^{2} u^{*}}{\partial y^{* 2}}=0 \text { at } y=a \\
& u^{*}=u_{\mathrm{p}}{ }^{*}=0, \mathrm{~T}^{*}=\mathrm{T}_{0}, \mathrm{C}^{*}=\mathrm{C}_{0}, \frac{\partial^{2} u^{*}}{\partial y^{* 2}}=0 \text { at } y=0
\end{aligned}
$$

The dusty fluid is assumed to be very thin with relatively low density. According to Ogulu and Bestman [10] the radiative heat flux is given by

$$
\frac{\partial q}{\partial y}=4 b^{2}\left(T_{0}-T\right)
$$

where $b$ is the mean radiation absorption coefficient.

To make the mathematical equations dimensionless, the following non- dimension variables are used.

$x=\frac{x^{*}}{a} ; \quad y=\frac{y^{*}}{a} ; \quad u=\frac{u^{*}}{U_{0}} ; \quad \operatorname{Re}=\frac{U_{0} a}{v} ; \quad t=\frac{t^{*} U_{0}}{a} ;$

$\operatorname{Pr}=\frac{v \rho C_{P}}{K^{*}} ; \theta=\frac{T^{*}-T_{0}}{T_{w}-T_{0}} ; \quad \varphi=\frac{C^{*}-C_{0}}{C_{w}-C_{0}} ; \quad H^{2}=\frac{\sigma B_{0}{ }^{2} a^{2}}{\rho v} ;$

$s^{2}=\frac{1}{D a} ; P=\frac{P^{*} a}{\rho v U_{0}} ; D a=\frac{k^{*}}{a^{2}} ; S c=\frac{D_{m}}{U_{0} a} ; \quad N^{2}=\frac{4 b^{2} a^{2}}{K^{*}} ;$

$\gamma^{2}=\frac{\eta}{v a^{2}} \quad ; \quad G c=\frac{g \beta^{*}\left(C_{w}-C_{0}\right) a^{2}}{v U_{0}} \quad ; \quad P e=\frac{U_{0} a \rho C_{P}}{K^{*}} ;$

$G r=\frac{g \beta\left(T_{w}-T_{0}\right) a^{2}}{v U_{0}} \quad ; \quad E=\frac{Q^{*} a^{2}}{K^{*}} ; K_{c}=\frac{K_{c}{ }^{{ }^{*}} a}{U_{0}} ;$

$M=\frac{v}{K_{0} a^{2}} ; \quad l=\frac{N_{0} K_{0} a^{2}}{\rho v} ;$

where $U_{0}$ is considered to be the mean flow velocity.

\section{Analytical Solution}

The dimensionless mathematical equations after the removal of stars can be written as

$\operatorname{Re} \frac{\partial u}{\partial t}=-\frac{\partial P}{\partial x}+\frac{\partial^{2} u}{\partial y^{2}}+\gamma^{2} \frac{\partial^{4} u}{\partial y^{4}}-\left(s^{2}+\frac{H^{2}}{1+m^{2}}+l\right) u+l u_{p}+(G r \theta+G c \varphi) \sin \alpha$

$-P^{\prime}(y)=0$

$\operatorname{Re} M u_{p}^{\prime}(t)=u-u_{p}$

$\operatorname{Re} \operatorname{Pr} \theta^{\prime}(t)=\theta^{\prime \prime}(y)+\left(N^{2}+E\right) \theta$

$\varphi^{\prime}(t)=S c \varphi^{\prime \prime}(y)-K_{C} \varphi$ 
Boundary conditions (6) and (7) become

$u=u_{\mathrm{p}}=0, \theta=1, \phi=1, u^{\prime \prime}(y)=0$ at $y=\mathrm{a}=1$

$u=u_{\mathrm{p}}=0, \theta=0, \phi=0, u^{\prime \prime}(y)=0$ at $y=\mathrm{a}=0$

Since the flow is oscillatory, we take

$$
\begin{gathered}
-\frac{\partial P}{\partial x}=\lambda e^{i \omega t} ; \quad u=u_{0} e^{i \omega t} \quad ; \quad u_{p}=u_{p_{0}} e^{i \omega t} ; \\
\theta=\theta_{0} e^{i \omega t} ; \quad \varphi=\varphi_{0} e^{i \omega t}
\end{gathered}
$$

where $\lambda$ is constant.

Putting Eq. (17) in Eqs. (10) to (16), we obtain

$$
\theta_{0}^{\prime \prime}(y)+m_{1}^{2} \theta_{0}=0
$$

$\varphi_{0}^{\prime \prime}(y)-m_{2}^{2} \varphi_{0}=0$

$\gamma^{2} u_{0}^{(i v)}(y)+u_{0}{ }^{\prime \prime}(y)-Z_{1} u_{0}=-\lambda-G r \theta_{0} \sin \alpha-G c \varphi_{0} \sin \alpha$

$u_{p_{0}}=\frac{u_{0}}{1+i \omega \operatorname{Re} M}$

The new wall conditions are given by

$u_{0}=u_{p_{0}}=0 ; \quad \theta_{0}=1 ; \phi_{0}=1 ; u^{\prime \prime}\left(y_{0}\right)=0$ on $y=\mathrm{a}=1$

$u_{0}=u_{p_{0}}=0 ; \quad \theta_{0}=1 ; \phi_{0}=0 ; u^{\prime \prime}\left(y_{0}\right)=0$ on $y=\mathrm{a}=0$

Differential equations (18 - 21) are evaluated using equations (22) and (23). The energy, species term and velocity equations of the dusty fluid are

$$
\begin{aligned}
& \theta(y, t)=\frac{\sin \left(m_{1} y\right)}{\sin \left(m_{1}\right)} e^{i \omega t} \\
& \phi(y, t)=\left(\frac{\sinh \left(m_{3} y\right)}{\sinh \left(m_{3}\right)}\right) e^{i \omega t}
\end{aligned}
$$

$u(y, t)=\left(\begin{array}{l}C_{1} e^{m_{3} y}+C_{2} e^{m_{4} y}+C_{3} e^{-m_{3} y}+C_{4} e^{-m_{4} y} \\ -Z_{2}-2 Z_{3} \sinh \left(m_{1} y\right)-2 Z_{4} \sinh \left(m_{2} y\right)\end{array}\right) e^{i \omega t}$

where the constants are given in the Appendix.

The velocity of the dust particles is given by

$$
u_{p}(y, t)=\frac{1}{1+i \omega \operatorname{Re} M}\left(\begin{array}{l}
C_{1} e^{m_{3} y}+C_{2} e^{m_{4} y}+C_{3} e^{-m_{3} y} \\
+C_{4} e^{-m_{4} y}-Z_{2}-2 Z_{3} \sinh \left(m_{1} y\right) \\
-2 Z_{4} \sinh \left(m_{2} y\right)
\end{array}\right) e^{i \omega t}
$$

\section{Shear Stress}

For dusty fluid, the friction between the fluid and the surface of dust particles moving at the top wall is given by

$$
\tau=-u^{\prime}(y) \text { at } y=a=1
$$

On simplification, we get

$\tau=-\frac{1}{1+i \omega \operatorname{Re} M}\left(\begin{array}{l}C_{1} m_{3} e^{m_{3} y}+C_{2} m_{4} e^{m_{4} y}-C_{3} m_{3} e^{-m_{3} y} \\ -C_{4} m_{4} e^{-m_{4} y}-2 Z_{3} m_{1} \cosh \left(m_{1} y\right) \\ -2 Z_{4} m_{2} \cosh \left(m_{2} y\right)\end{array}\right) e^{i \omega t}$

\section{Nusselt Number}

The heat conduction at the top wall is

$$
\begin{aligned}
& N u=-\theta^{\prime}(y) a t \quad y=a=1 \\
& N u=-\frac{m_{1} \cos \left(m_{1} y\right)}{\sin \left(m_{1}\right)} e^{i \omega t} \text { at } y=1
\end{aligned}
$$

\section{Sherwood Number}

The mass conduction at the top wall is

$$
\begin{aligned}
& S h=-\varphi^{\prime}(y) \text { at } \quad y=a=1 \\
& S h=-\left(\frac{m_{3} \cosh \left(m_{3} y\right)}{\sinh \left(m_{3}\right)}\right) e^{i \omega t} \text { at } y=1
\end{aligned}
$$

\section{Plotting Graphs and Discussions}

We ignore the imaginary part and consider only the real part of the results obtained in equations (24) $-(31)$. The following parameter values $P e=0.71, G r=1, G c=1, K c=1, S c=1, \operatorname{Re}=1, t=0, w$ $=1, s=1, H=1, N=1, \alpha=\pi / 4, m=1, B=1$ and $\gamma=1$ are used. The above values are kept common in the entire study and the other quantities are varied. The corresponding graphs are plotted. (Figures 2 to 8 )

Fig. 2 shows the effect of Peclet number on velocity profile. Higher estimations of Pe shows the higher values of velocity.

Fig. 3 The thermal effect or heat radiation parameter $(\mathrm{N})$ shows a remarkable role; i.e. the increase of $\mathrm{N}$ shows a velocity increase throughout the middle of the channel and is maximum at the midpoint. It increases near lower wall whereas it decreases near the top wall.

Fig. 4 shows the effect of Grashoff number $(\mathrm{Gr})$ on velocity profile. When $\mathrm{Gr}>1.0$, the maximum velocity is obtained at $\mathrm{y}<0.5$ i.e at the lower part of the channel.

From Fig. 5, it is clear that the presence of Hall current $(\mathrm{m}>0)$, the velocity is always positive for all external magnetic field. When $\mathrm{H}>9.3$ and $\mathrm{m}=0$, we get negative velocity.

It is necessary to observe the limit of magnetic field for continuous application of it. $\mathrm{B}_{\mathrm{c}}$ is the critical magnetic field at which the velocity becomes zero. It is evident that from Fig. 6 , that the forward movement of the fluid beyond $\mathrm{B}_{\mathrm{c}}$ is possible only because of the presence of Hall current.

Fig. 7 displays the increase in radiation parameter diminishes the temperature effect.

From Fig. 8, we understand that the concentration does not affect much due to the rise in the value of Sc.

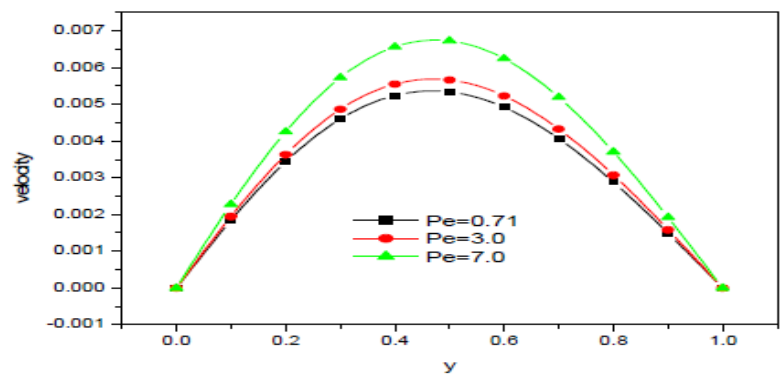

Fig. 2: Comparison of Velocity with Pe 


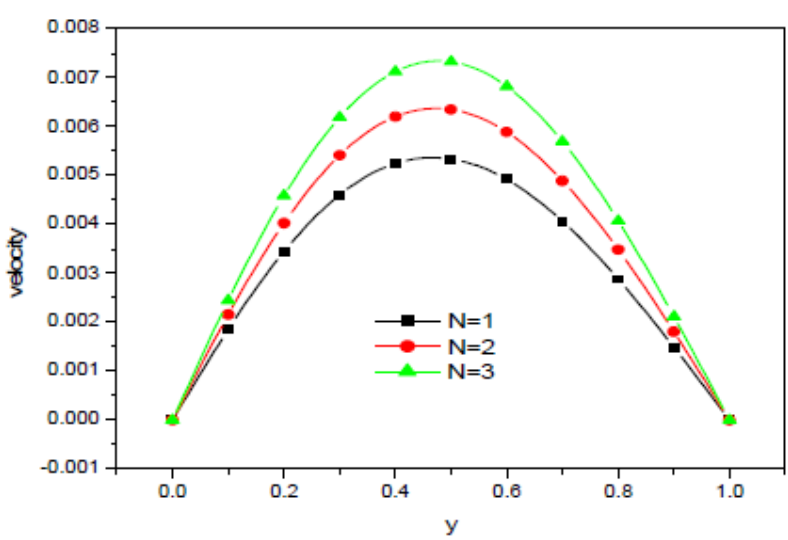

Fig. 3: Comparison of Velocity with $\mathbf{N}$

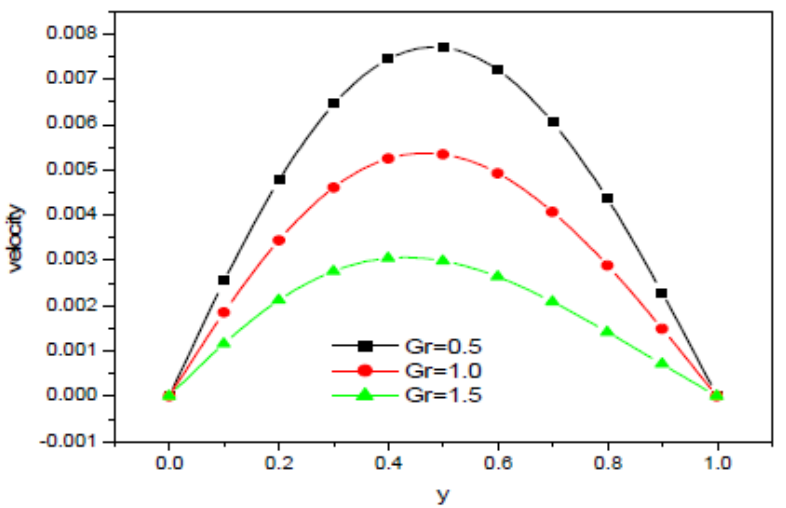

Fig. 4: Comparison of Velocity with $\mathbf{G r}$
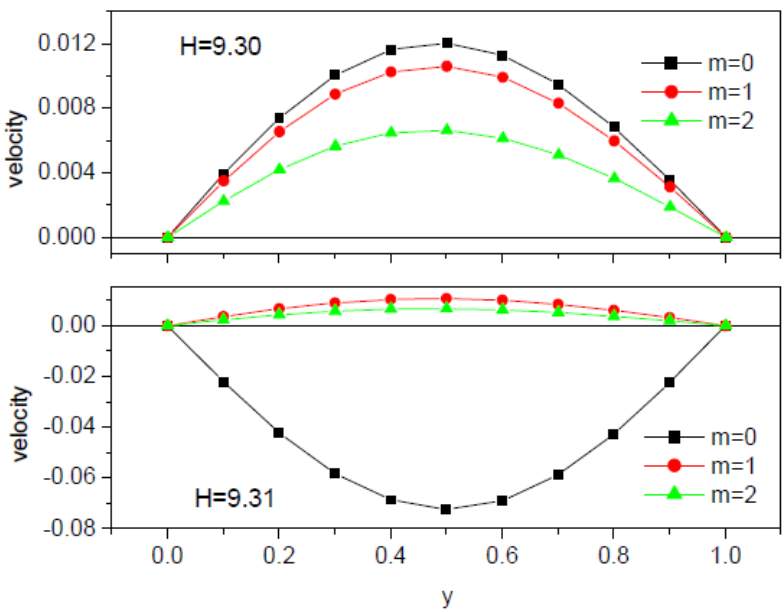

Fig. 5: Limit of External Magnetic field for the positive fluid velocity

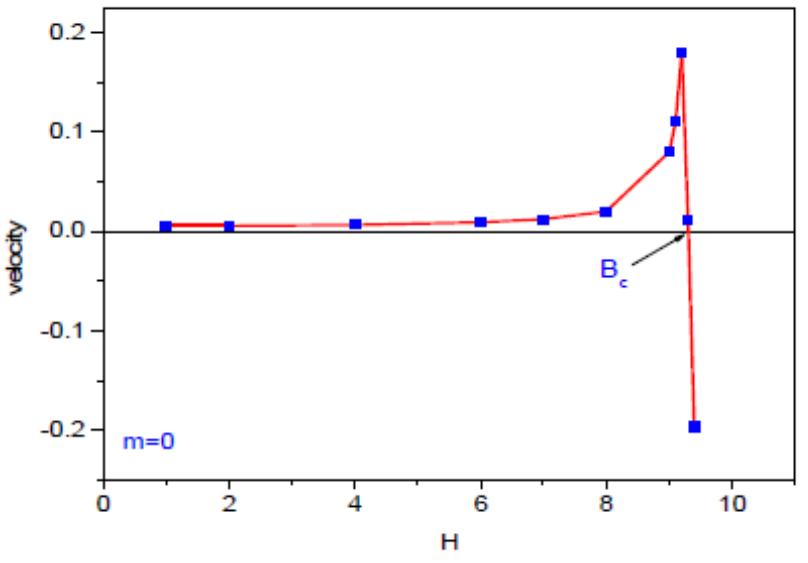

Fig. 6: Determination of critical magnetic field $\mathrm{B}_{\mathrm{C}}$

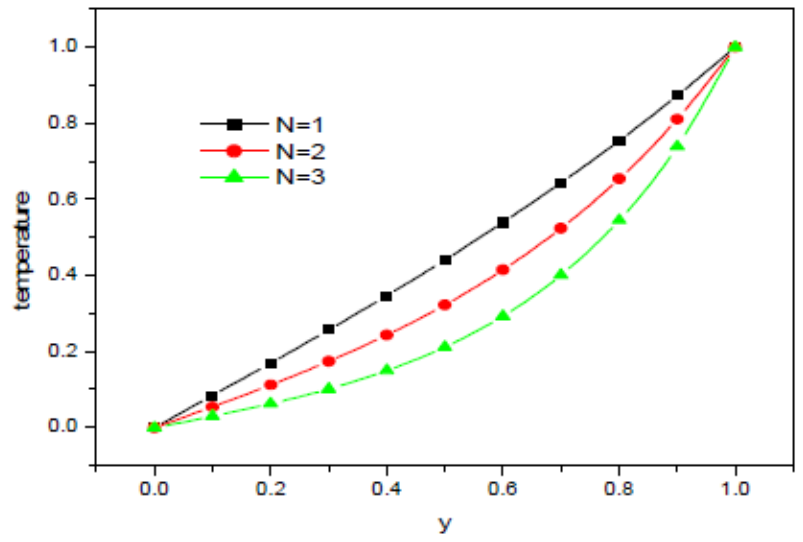

Fig. 7: Temperature varies for different values of $\mathrm{N}$

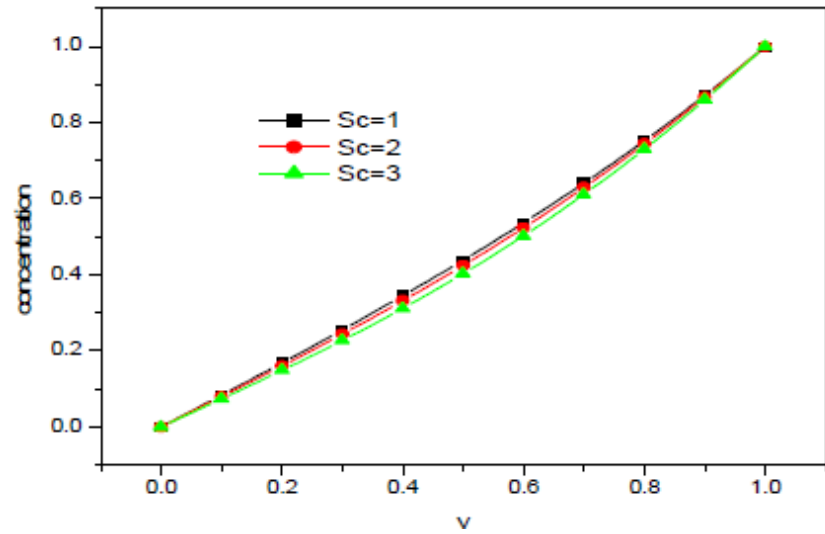

Fig. 8: Concentration differs for different values of Sc

\section{Conclusion}

This paper investigates the effect of hall current in MHD oscillatory flow of a couple stress dusty fluid in an inclined channel. The following findings are obtained for this work.

- $\quad$ Higher values of Pe, $\mathrm{N}$ shows the higher values of velocity.

- $\quad$ The velocity profile decreases due to an increase in Gr.

- A negative velocity is expected beyond $\mathrm{H}=9.3$, if there is no Hall current.

- The critical magnetic field's limit is measured for forward direction of the fluid. The Hall current effect can nullify the effect of $\mathrm{B}_{\mathrm{C}}$

- $\quad$ The temperature profile decreases as $\mathrm{N}$ increases.

- The concentration profile decreases as Sc increases.

\section{Nomenclature}

$(x, y) \quad x$ and $y$ coordinates along $\mathrm{X}, \mathrm{Y}$ axis

$u \quad$ velocity component along $x$ direction

$t \quad$ dimensional time

$T$ fluid temperature

$P \quad$ dimensional pressure

$\mathrm{C}$ fluid concentration

$\mathrm{B}_{0}=\mu_{\mathrm{e}} \mathrm{H}_{0}$ electromagnetic induction

$g \quad$ gravitational force

$m \quad$ Hall current parameter

$H \quad$ Hartmann number

Da Darcy

$\mathrm{Pe} \quad$ Peclet number

$\mathrm{C}_{\mathrm{P}} \quad$ fluid's specific heat at constant pressure

Sc Schmidt number

$\mathrm{Gr} \quad$ Grashof number for heat transfer

$\mathrm{Nu} \quad$ Nusselt number 
Gc modified Grashof number

Re Reynolds number

$N \quad$ thermal radiation parameter

Sh Sherwood number

$\mathrm{K}_{\mathrm{C}} \quad$ chemical reaction parameter

$q \quad$ heat flux term for radiation

$s \quad$ permebility factor

$\mathrm{D}_{\mathrm{m}} \quad$ mass diffusion coefficient

$\mathrm{C}_{f} \quad$ shear stres

Q dimensional heat source parameter

E dimensionless heat source parameter

$\mathrm{T}_{0}, \mathrm{~T}_{w} \quad$ Lower and upper wall temperature

$\mathrm{C}_{0}, \mathrm{C}_{w} \quad$ Lower and upper wall concentration

\section{Greek Symbols}

\section{$\lambda \quad$ wavelength}

$\alpha \quad$ Angle of inclination

$\varphi \quad$ phase difference

$\eta \quad$ couple stress coefficient

$\sigma \quad$ fluid conductivity

$\rho \quad$ density of fluid

$v \quad$ kinematic viscosity

$\tau \quad$ skin friction

$\theta$ dimensionless fluid temperature function

$\omega \quad$ angular frequency of the oscillation

$\gamma \quad$ couple stress parameter

$\mu_{e} \quad$ magnetic permeability (Dynamic viscosity)

$\phi \quad$ mass concentration profile

$\delta_{\mathrm{C}} \quad$ concentration variation parameter

$\delta_{\mathrm{T}} \quad$ temperature variation parameter

$\beta \quad$ temperature's thermal expansion coefficient

$\beta^{*} \quad$ concentration's thermal expansion coefficient

\section{Appendix}

$$
\begin{aligned}
& m_{1}=\sqrt{N^{2}+E-i \omega \operatorname{RePr}} \quad m_{2}=\sqrt{\frac{K_{c}+i \omega}{S c}} \\
& Z_{1}=\sqrt{s^{2}+\frac{H^{2}}{1+m^{2}}+l+i \omega \operatorname{Re}-\frac{l}{1+i \omega \operatorname{Re} M}} \\
& m_{3}=\sqrt{\frac{-1+\sqrt{-1+4 Z_{1} \gamma^{2}}}{2 \gamma^{2}}} \\
& m_{4}=\sqrt{\frac{-1-\sqrt{-1+4 Z_{1} \gamma^{2}}}{2 \gamma^{2}}} \\
& A_{1}=\gamma^{2} m_{i}^{4}+m_{i}^{2}-Z_{1} ; i=1,2 \\
& Z_{2}=\frac{\lambda}{Z_{1}} \\
& Z_{3}=\frac{G r \sin \alpha}{2 A_{1} \sinh m_{1}} \\
& Z_{5}=2 Z_{3} \sinh m_{1} \\
& Z_{7}=2 Z_{3} m_{1}^{2} \sinh m_{1} \\
& C_{1}=-C_{2}-C_{3}-C_{4}+Z_{2} \\
& Z_{4}=\frac{G c \sin \alpha}{2 A_{2} \sinh m_{2}} \\
& Z_{6}=2 Z_{4} \sinh m_{2} \\
& C_{3}=-\frac{1}{2 \sinh m_{3}}\left[C_{2}\left(e^{m_{3}}-e^{m_{4}}\right)+C_{4}\left(e^{m_{3}}-e^{-m_{4}}\right)-Z_{2}\left(e^{m_{3}}-1\right)+\left(Z_{5}+Z_{6}\right)\right] \\
& C_{4}=-\frac{Z_{2} m_{3}{ }^{2}\left(1-e^{m_{4}}\right)+\left(Z_{5}+Z_{6}\right) m_{3}{ }^{2}-\left(Z_{7}+Z_{8}\right)}{\left(m_{3}{ }^{2}-m_{4}{ }^{2}\right) 2 \sinh m_{4}}
\end{aligned}
$$

\section{References}

[1] Saffman P G, On the stability of laminar flow of a dusty gas, J. of Fluid Mech. Vol. 13, 1962, pp 120-128.

[2] Gabriela Varvara, Florin Topoliceanu, Dan Zaharia, The Blood Two-phase Dusty fluid flow modelling, IFAC Modelling and Control in Biomedical Systems, Warwick, UK, 1997.

[3] D Makinde, P Y Mhone, Heat Transfer to MHD Oscillatory Flow in a Channel filled with permeable medium, Rom. Journ. Phys. , Vol. 50, 2005, pp 931-938.

[4] V. K. Stokes, Couple stresses in fluids, Vol. 9, 1996, pp 1709-1715.

[5] Kulshretha P K and Puri P, Wave structure in oscillatory Couette flow of a dusty gas, Acta Mech. Springer Verlag, Vol. 46, pp 127 128.

[6] Gireesha B J, Roopa G S and Bagewadi C S, Unsteady flow and heat transfer of a dusty fluid through a rectangular channel, Mathematical Problems in Engineering Hindawi Publishing Corporation.

[7] Makinde O D and Chinyoka T, MHD transient flows and heat transfer of dusty fluid in a channel with variable physical properties and Navier slip condition, Comput. Math. Appl., Vol. 60 pp 660669.

[8] Prakash O Kumar D and Dwivedi Y K, MHD free convection flow of a visco-elastic dusty gas through a semi infinite plate moving with velocity decreasing exponentially with time and radiative heat transfer AIP 2011

[9] R Vijayalakshmi, A Govindarajan, J Sasikumar, E P Siva, Chemical reaction effects on radiative MHD oscillatory flow in a permeable channel with mass transfer and heat absorption Int. J. of Scientific \& Engineering Research Vol. 6 Issue 3 March 2015.

[10] M Vidhya, R Vijayalakshmi, A Govindarajan, Chemical reaction effects on radiative MHD oscillatory flow in a permeable channel with heat and mass transfer in an asymmetric channel ARPN Journal of Engg. And Applied Sciences Vol. 10 No. 4 March 2015.

[11] R. Vijayalakshmi, A. Govindarajan, Slip effect on radiative MHD oscillatory flow with chemical reaction in an asymmetric channel, Global Journal of Pure and Applied Mathematics Vol. 12 No. 2 (2016)

[12] R. Vijayalakshmi, A. Govindarajan, Chemically reacting MHD oscillatory slip flow in an asymmetric channel with both concentration and temperature variation, International Journal of Pure and Applied Mathematics, Vol. 114, No. 6, 2017, pp 61 - 66.

[13] Om Prakash O D Makinde Heat transfer to MHD oscillatory dusty fluid flow in a channel filled with a permeable medium Sadhana Vol 40 June 2015 pp 1273-1282.

[14] A Ogulu A R Bestman, Deep Heat Muscle Treatment - A Mathematical Model pp 3-16.

[15] Govindarajan, R. Vijayalakshmi, V. Ramamurthy, Combined effects of heat and mass transfer to magneto hydrodynamics oscillatory dusty fluid flow in a permeable channel, Journal of Physics: Conference Series 1000, 2018

[16] Nirmala P. Ratchagar, V. Balakrishnan and R. Vasanthakumari, Effect of Hall current in oscillatory flow of a couple stress fluid in an inclined channel, Annals of Pure and Applied Mathematics, Vol. 16,2018 , pp $151-169$

[17] M. Thamizhsudar, J. Pandurangan, Hall effects and rotation effects on MHD flow past an exponentially accelerated vertical plate with combined heat and mass transfer effects, Int. J. of Applied Mechanics and Engineering, Vol. 20, No. 3, 2015, pp $605-616$.

[18] Dulal Pal, Babulal Talukdar, Influence of Hall current and Thermal radiation on MHD convective heat and mass transfer in a rotating permeable channel with chemical reaction, International Journal of Engineering Mathematics, Vol. 2013.

[19] K. D. Singh, Khem Chand and Shavnam Sharma, Hydromagnetic oscillatory flow of dusty fluid in a rotating permeable channel, International J. of Mathematical Archive, 2012, pp 2331 - 2339.

[20] Khem Chand, Sanjeev Kumar, Effect of Hall current and rotation on chemically reacting and radiating MHD oscillatory dusty viscoelastic flow through permeable vertical channel, Int. J. of Applied Mathematics and Computation, Vol. 5, 2013, pp 17-32. 